\section{A Practical Guide to Experimentation (and Benchmarking)}

Nikolaus Hansen

Research Centre Saclay, CMAP, Ecole polytechnique

Permission to make digital or had copies of part or all of this work for personal

or classroom use is granted without fee provided that copies are not made or
distributed for por

and the full citation on the first page. Copyrights for third-party components of

GECCO '19 Companion, July 13-17, 2019, Prague, Czech Republic

O 2019 Copyright is held by the owner/author(s).

hittrs://doi org/10 1145/33196193323397

\section{Why Experimentation?}

- The behaviour of many if not most interesting algorithms is

- not amenable to a (full) theoretical analysis even when applied to simple problems

calling for an alternative to theory for investigation

- not fully comprehensible or even predictable without (extensive) empirical examinations comprehension is the main driving force for scientific progress evroblems If it disagrees with experiment, it's wrong. And that simple statement is the key to science. - R. Feynman

- Virtually all algorithms have parameters

like most (physical/biological/...) models in science we rarely have explicit knowledge about the "right" choice this is a big obstacle in designing and benchmarking algorithms

- We are interested in solving black-box optimisation problems which may be "arbitrarily" complex and (by definition) not well-understood

\section{Overview}

- Scientific experimentation

- Invariance

- Statistical Analysis

- A practical experimentation session

- Approaching an unknown problem

- Performance Assessment

- What to measure

- How to display

- Aggregation

- Empirical distributions

Do not hesitate to ask questions!

\section{Scientific Experimentation (dos and don'ts)}

- What is the aim? Answer a question, ideally quickly and comprehensively

consider in advance what the question is and in which way the experiment can answer the question

What are the dos and don'ts?

- what is most helpful to do?

- what is better to avoid? 


\section{Scientific Experimentation (dos and don'ts)}

What is the aim? Answer a question, ideally quickly (minutes, seconds) and comprehensively

consider in advance what the question is and in which way the experiment can answer the question

- do not (blindly) trust in what one needs to rely upon (code, claims, ...) without good reasons

check/test "everything" yourself, practice stress testing (e.g. weird parameter setting) which also boosts understanding interpreted/scripted languages have an advantage Why Most Published Research Findings Are False [loannidis 2005]

- practice to make predictions of the (possible/expected) outcome(s)

$$
\text { to develop a mental model of the object of interest }
$$

to practice being proven wrong, to overcome confirmation bias

- run rather many than few experiments iteratively, practice online experimentation (see demonstration) to run many experiments they must be quick to implement and run, develops a feeling for the effect of setup changes

\section{Scientific Experimentation (dos and don'ts)}

- don't make minimising CPU-time a primary objective avoid spending time in implementation details to tweak performance yet code optimization may be necessary to run experiments efficiently

\section{Testing Heuristics: We Have it All Wrong [Hooker 1995]}

"The emphasis on competition is fundamentally anti-intellectual and does not build the sort of insight that in the long run is conducive to more effective algorithms"

- It is usually (much) more important to understand why algorithm A performs badly on function $f$, than to make algorithm A faster for unknown, unclear or trivial reasons

mainly because an algorithm is applied to unknown functions, not to $f$, and the "why" allows to predict the effect of design changes

- there are many devils in the details, results or their interpretation may crucially depend on simple or intricate bugs or subtleties yet another reason to run many (slightly) different experiments check limit settings to give consistent results

\section{Scientific Experimentation (dos and don'ts)}

- run rather many than few experiments iteratively, practice online experimentation (see demonstration)

to run many experiments they must be quick to implement and run, ideally seconds rather than minutes (start with small dimension/budget) develops a feeling for the effect of setup changes

run any experiment at least twice

assuming that the outcome is stochastic get an estimator of variation/dispersion/variance

- display: the more the better, the better the better

figures are intuition pumps (not only for presentation or publication) it is hardly possible to overestimate the value of a good figure data is the only way experimentation can help to answer questions, therefore look at them, study them carefully!

- don't make minimising CPU-time a primary objective

avoid spending time in implementation details to tweak performance prioritize code clarity (minimize time to change code, to debug code, to maintain code) yet code optimization may be necessary to run experiments efficiently

\section{Scientific Experimentation (dos and don'ts)}

- there are many devils in the details, results or their interpretation may crucially depend on simple or intricate bugs or subtleties yet another reason to run many (slightly) different experiments check limit settings to give consistent results

- Invariance is a very powerful, almost indispensable tool 


\section{Invariance: binary variables}

Assigning 0/1 (for example minimize $\sum_{i} x_{i}$ vs $\sum_{i} 1-x_{i}$ )

- is an "arbitrary" and "trivial" encoding choice and

- amounts to the affine linear transformation $x_{i} \mapsto-x_{i}+1$

this transformation or the identity are the coding choice in each variable in continuous domain: norm-preserving (isotropic, "rigid") transformation

- does not change the function "structure"

- all level sets $\{x \mid f(x)=$ const $\}$ have the same size (number of elements, same volume)

- the same neighbourhood

- no variable dependencies are introduced (or removed)

Instead of 1 function, we now consider $2^{\star *} n$ different but equivalent functions $2^{\star *} n$ is non-trivial, it is the size of the search space itself

\section{Invariance Under Order Preserving Transformations}
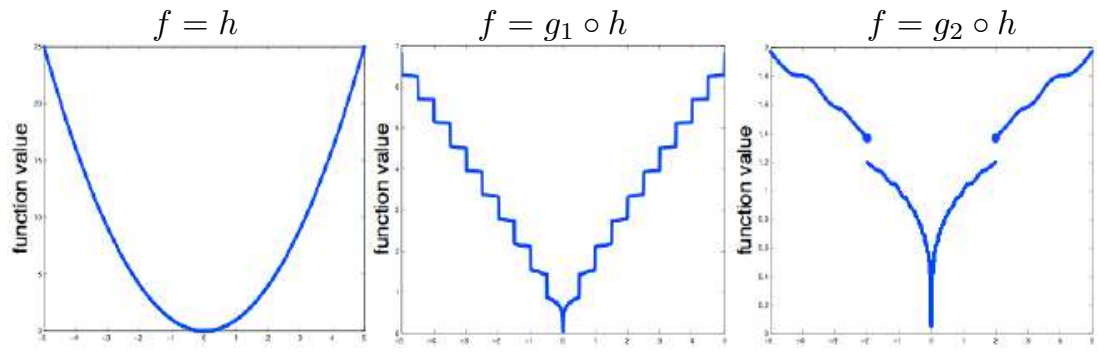

Three functions belonging to the same equivalence class

A function-value free search algorithm is invariant under the transformation with any order preserving (strictly increasing) $g$.

\section{Invariances make}

- observations meaningful

as a rigorous notion of generalization

- algorithms predictable and/or "robust"

\section{Invariance: binary variables}

Permutation of variables

- is another "arbitrary" and "trivial" encoding choice and

- is another norm-preserving transformation

- does not change the function "structure" (as above)

- may affect the neighbourhood depending on the operators (recombination, mutation)

Instead of 1 function, we now consider $n$ ! different but equivalent functions

$n ! \gg 2^{n} \quad$ is much larger than the size of the search space

\section{Invariance Under Rigid Search Space Transformations}

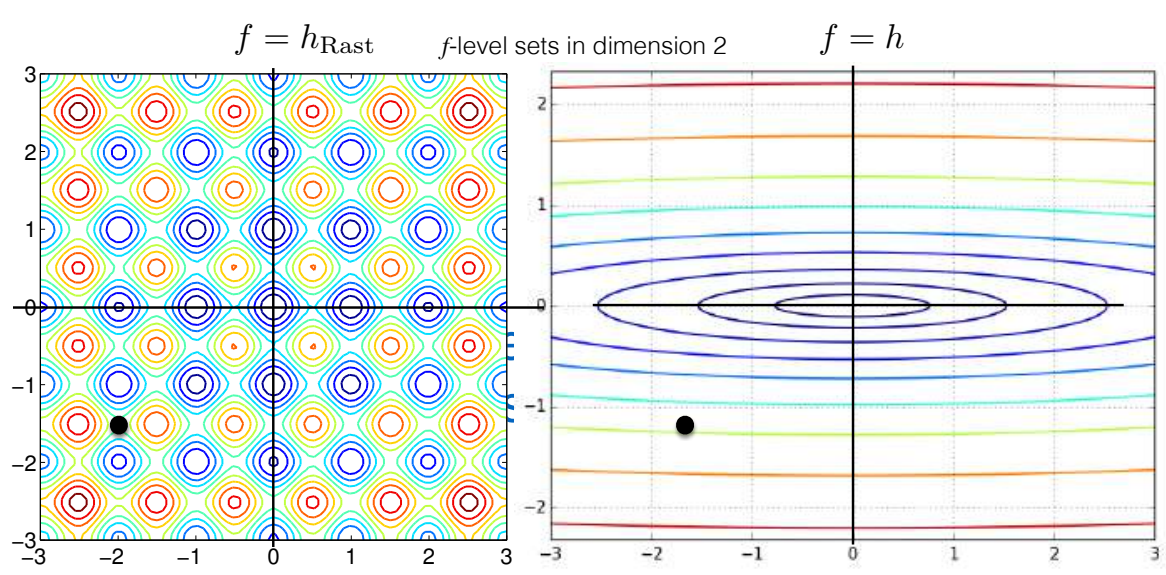

for example, invariance under search space rotation (separable vs non-separable) 
Invariance Under Rigid Search Space Transformations

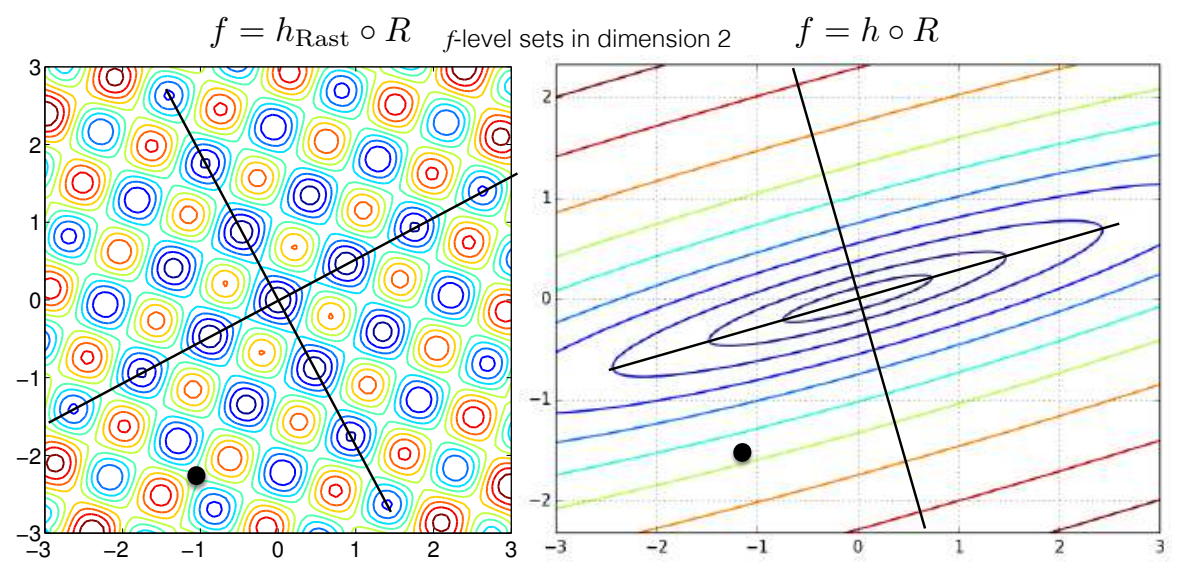

for example, invariance under search space rotation (separable vs non-separable)

\section{Measuring Performance}

\section{Empirically}

convergence graphs is all we have to start with

the right presentation is important!

\section{Invariance}

The grand aim of all science is to cover the greatest number of empirical facts by logical deduction from the smallest number of hypotheses or axioms. - Albert Einstein

- Empirical performance results

- from benchmark functions

- from solved real world problems

are only useful if they do generalize to other problems

- Invariance is a strong non-empirical statement about generalization

generalizing (identical) performance from a single function to a whole class of functions

Consequently, invariance is of greatest importance for the assessment of search algorithms.

\section{Displaying Three Runs}

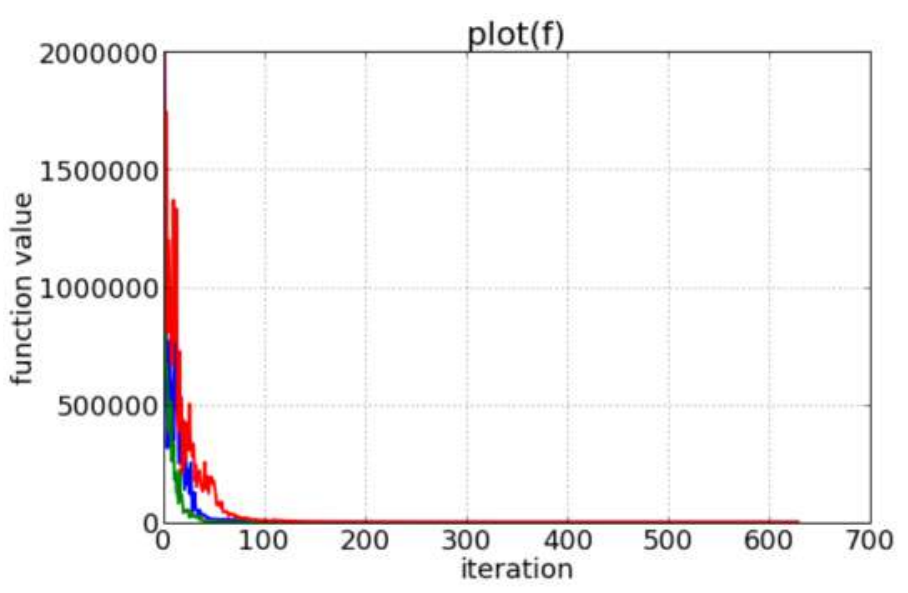

not like this (it's unfortunately not an uncommon picture)

why not, what's wrong with it? 

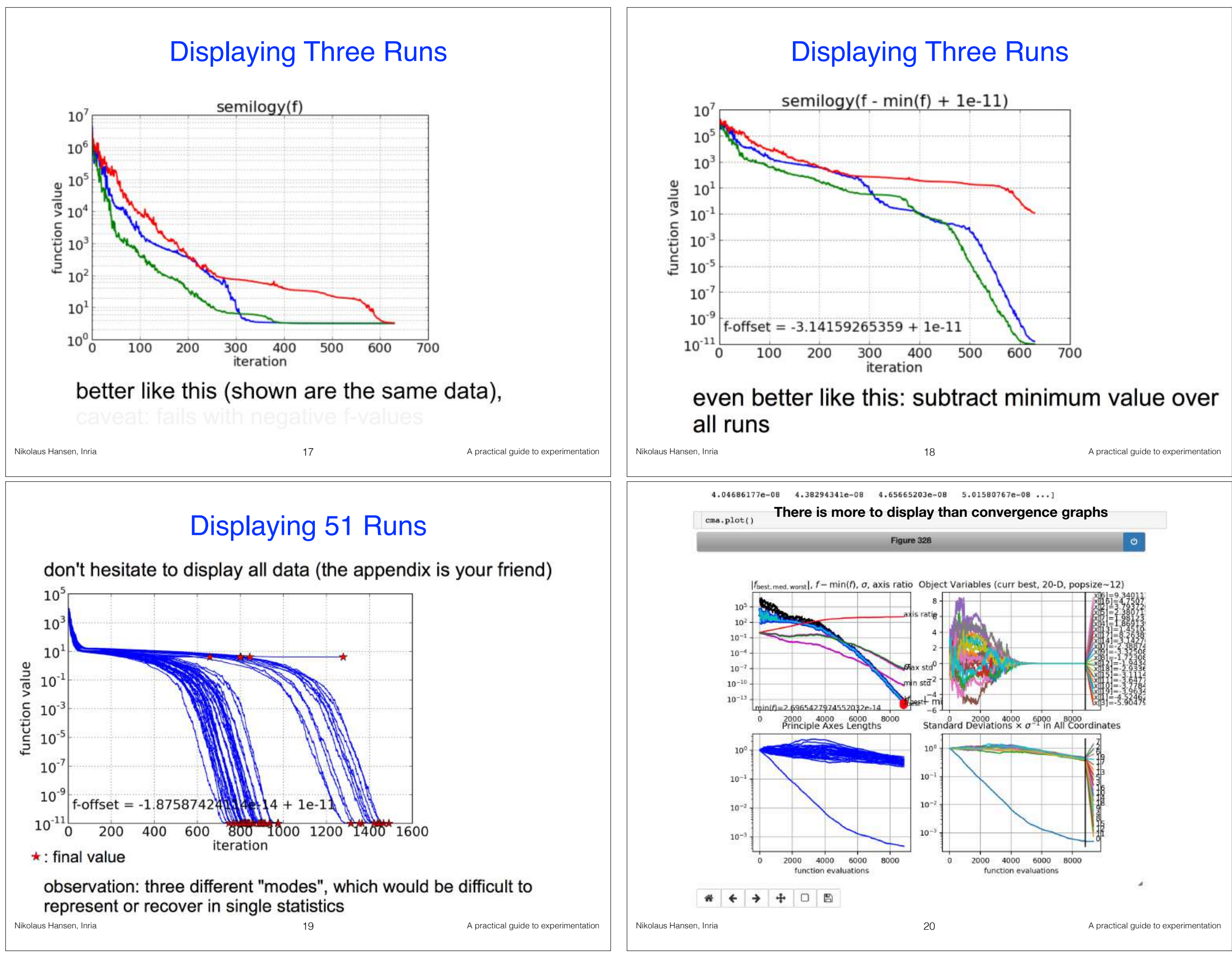


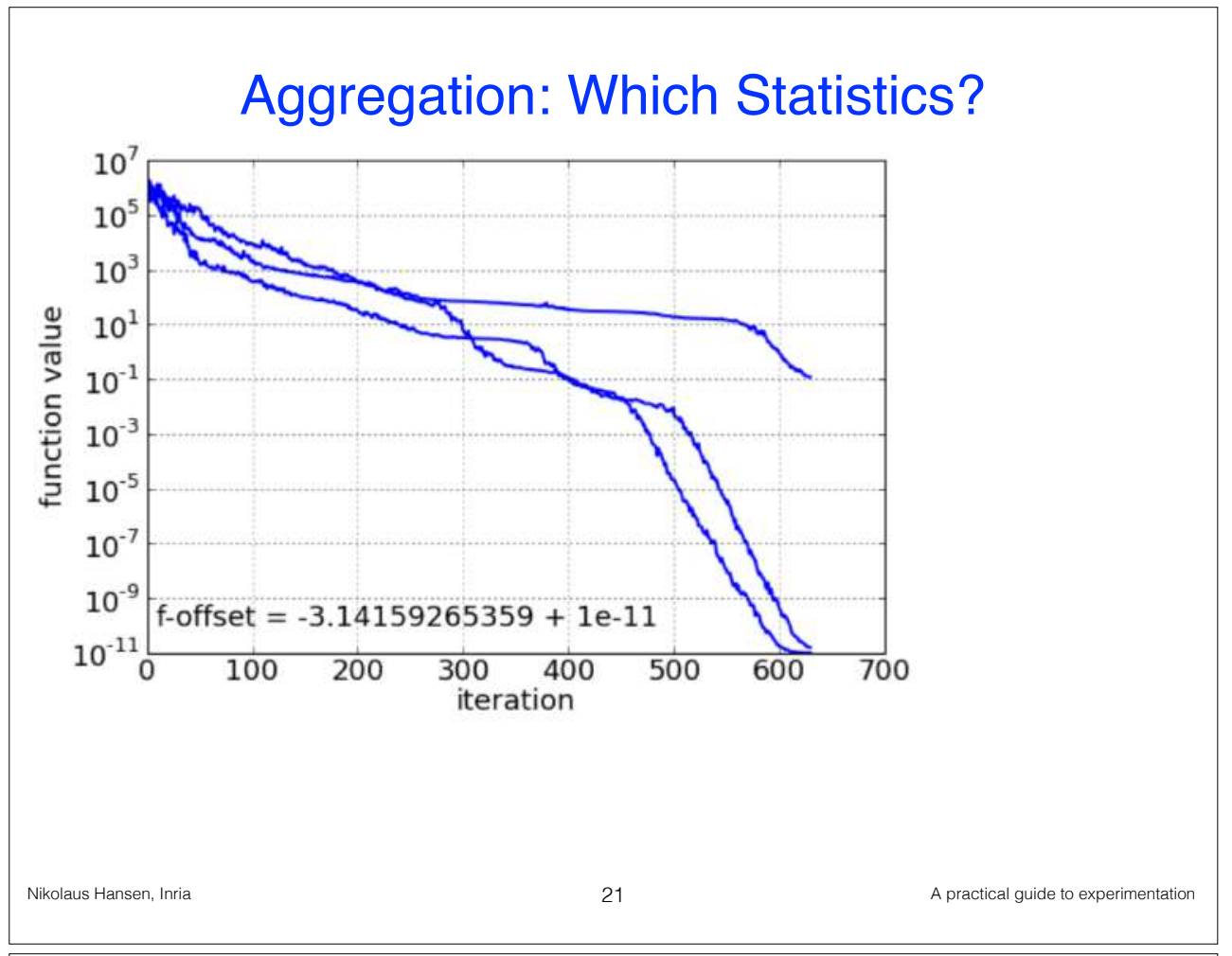

\section{More Caveats on Averages/Expectations}

- to reliably estimate an expectation (from the average) we need to make assumptions on the tail of the underlying distribution

- these can not be implied from the observed data

- AKA: the average is well-known to be (highly) sensitive to outliers (extreme events)

- rare events can only be analyzed by collecting a large enough number of data

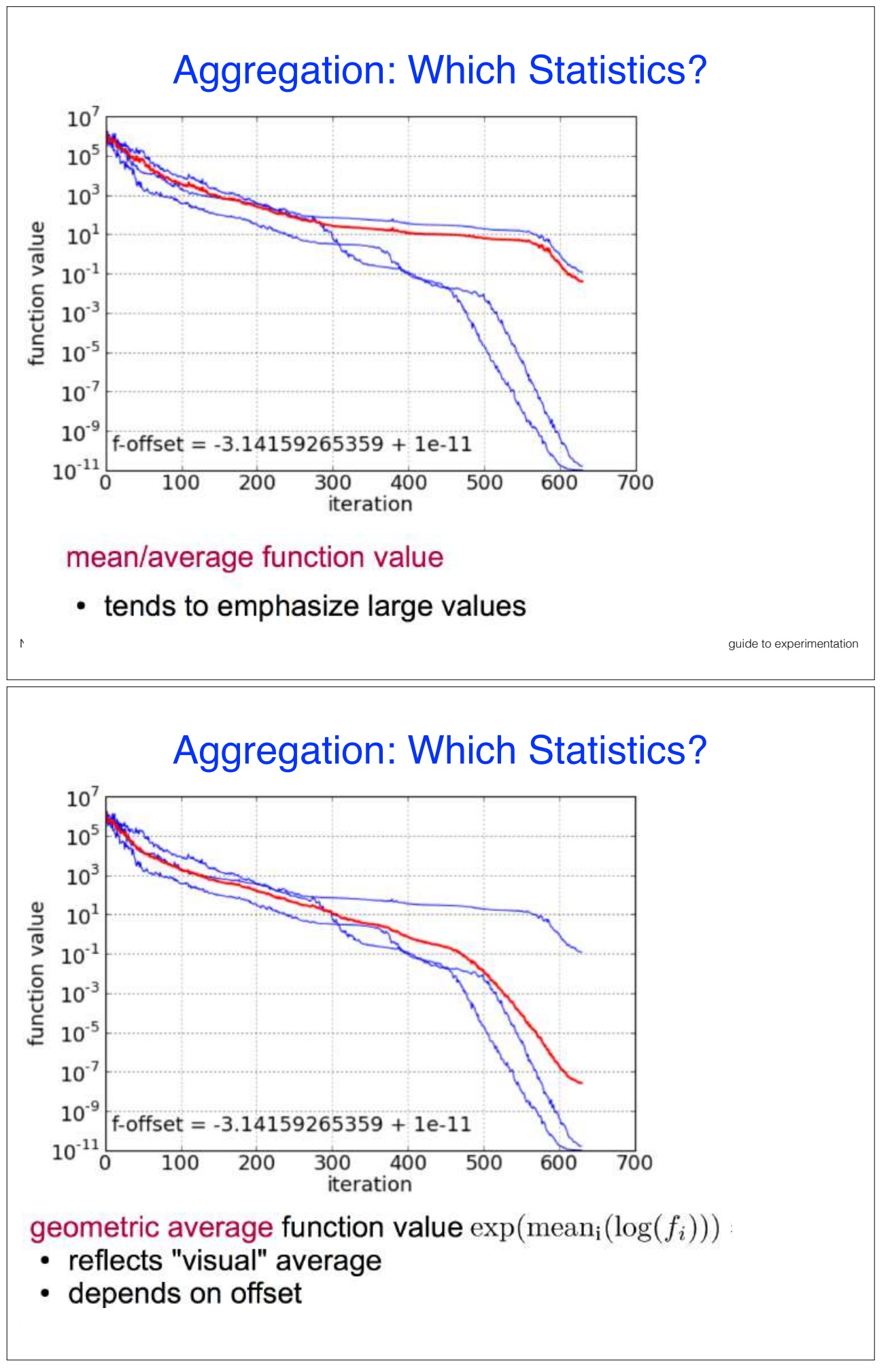




\section{Aggregation: Which Statistics?}

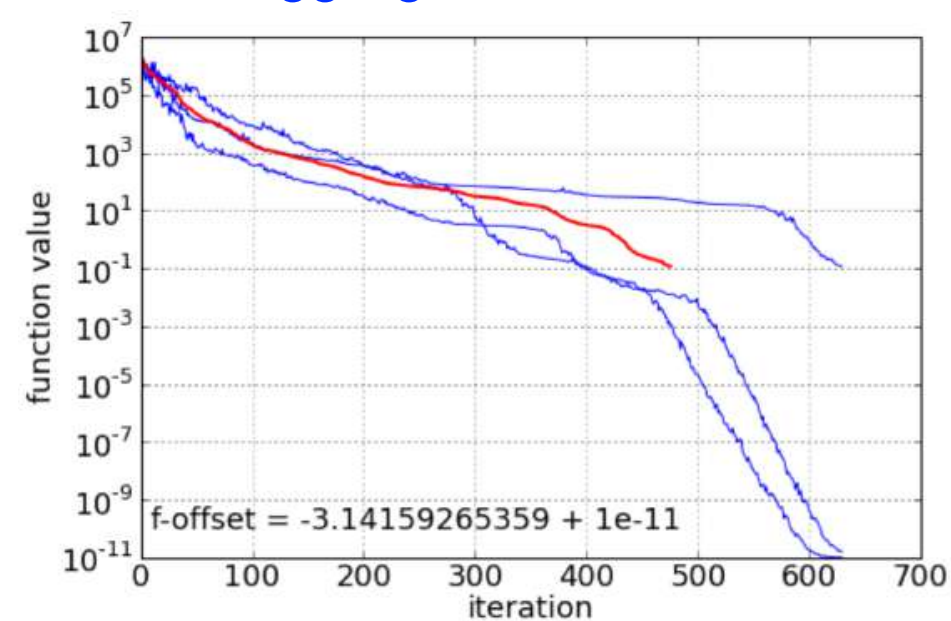

average iterations

- reflects "visual" average

- here: incomplete

\section{Implications}

- use the median as summary datum

unless there are good reasons for a different statistics out of practicality: use an odd number of repetitions

- more general: use quantiles as summary data

for example out of 15 data: 2nd, 8th, and 14th value represent the $10 \%, 50 \%$, and $90 \%$-tile

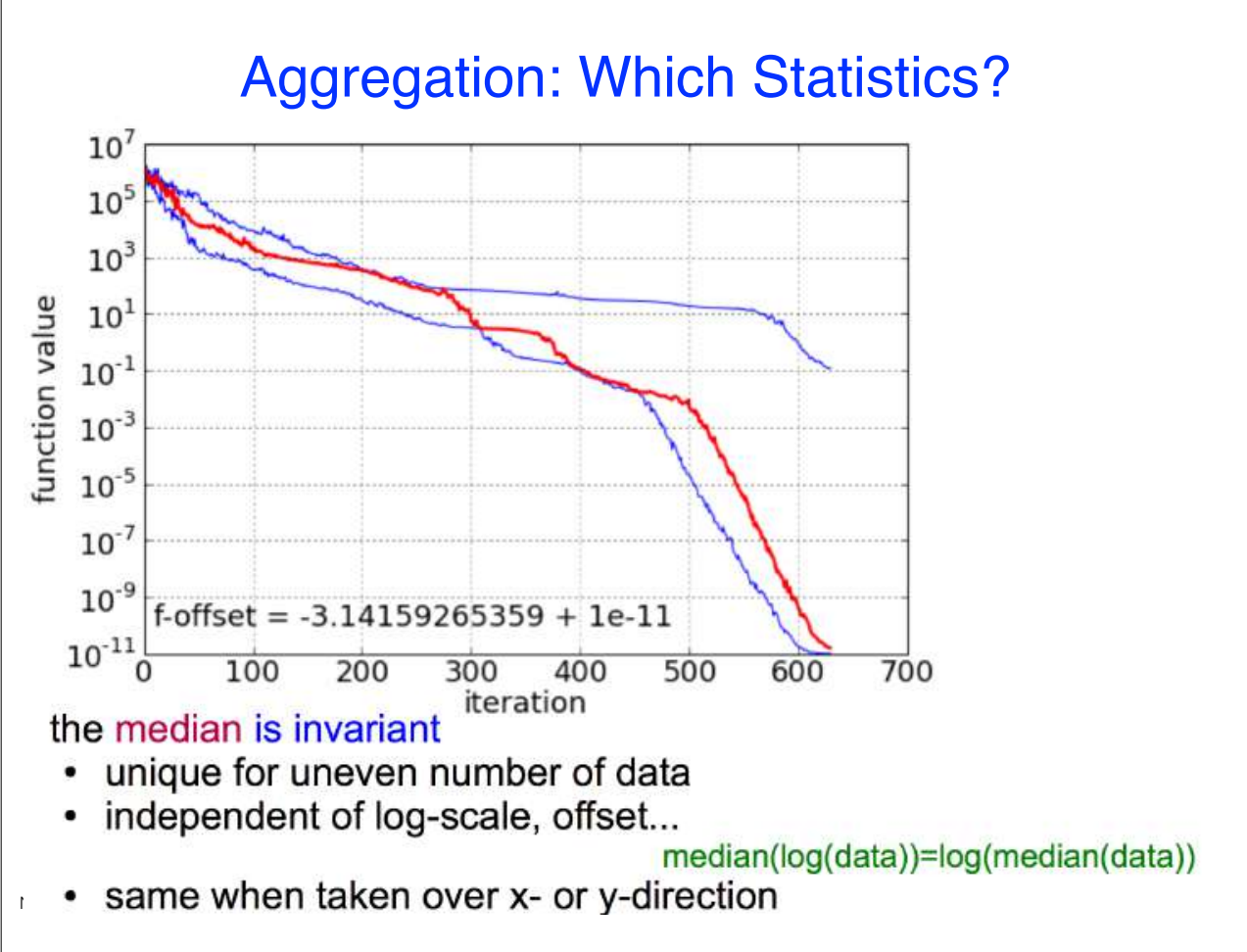

\section{Two More Examples}
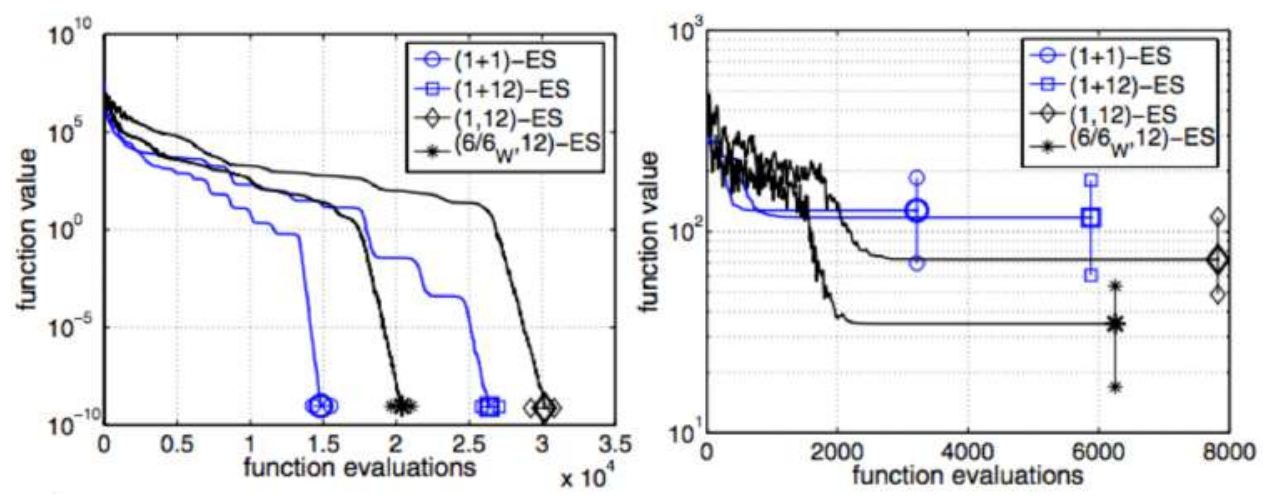

Comparison of 4 algorithms using the "median run" and the $90 \%$ central range of the final value on two different functions (Ellipsoid and Rastrigin)

caveat: this range display with simple error bars fails, if, e.g., $30 \%$ of all runs "converge" 


\section{Statistical Analysis}

"The first principle is that you must not fool yourself, and you are the easiest person to fool. So you have to be very careful about that. After you've not fooled yourself, it's easy not to fool other[ scientist]s. You just have to be honest in a conventional way after that."

- Richard P. Feynman

\section{Rare Events}

- The obvious: if we consider rare events to be important, we have to sample many data

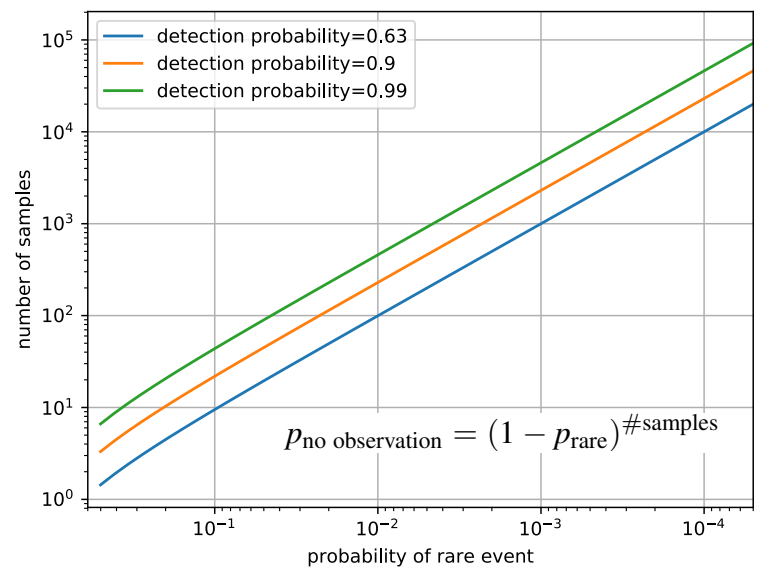

\section{Statistical Analysis}

"experimental results lacking proper statistical analysis must be considered anecdotal at best, or even wholly inaccurate"

- M. Wineberg

Do you agree (sounds about right) or disagree (is taken a little over the top) with the quote?

an experimental result (shown are all data obtained):

Do we (even) need a statistical analysis?

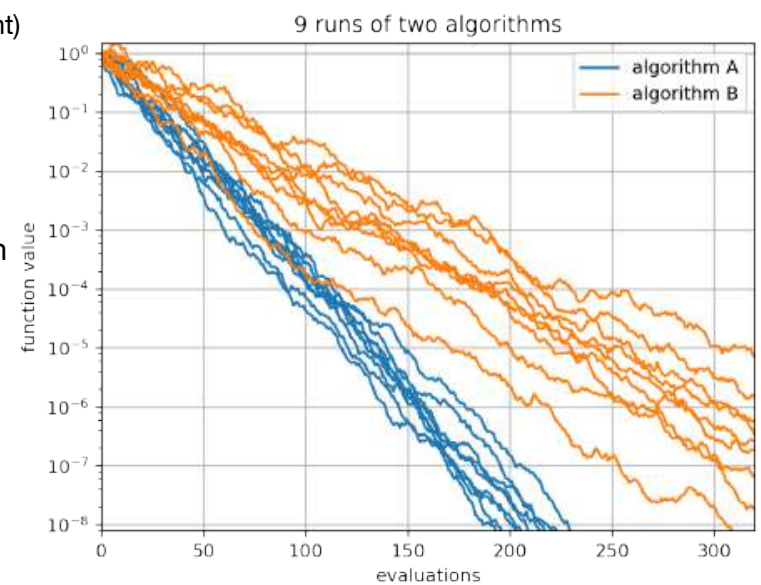

Nikolaus Hansen, Inria

\section{Statistical Significance: General Prodecure}

first, check the relevance of the result, for example of the difference which is to be tested for statistical significance this also means: do not explorative testing (e.g. test all pairwise combinations) any ever so small difference can be made statistically significant with a simple trick, but not made significant in the sense of important or meaningful

- prefer "nonparametric" methods

not assuming that the data come from a parametrised family of probability distributions

- $p$-value $=$ significance level $=$ probability of a false positive outcome, given $\mathrm{HO}$ is true

smaller $p$-values are better $<0.1 \%$ or $<1 \%$ or $<5 \%$ is usually considered as statistically significant

- given a found/observed p-value, fewer data are better

more data (almost inevitably) lead to smaller $p$-values, hence to achieve the same $\mathrm{p}$-value with fewer data, the between-difference must be larger compared to the within-variation 


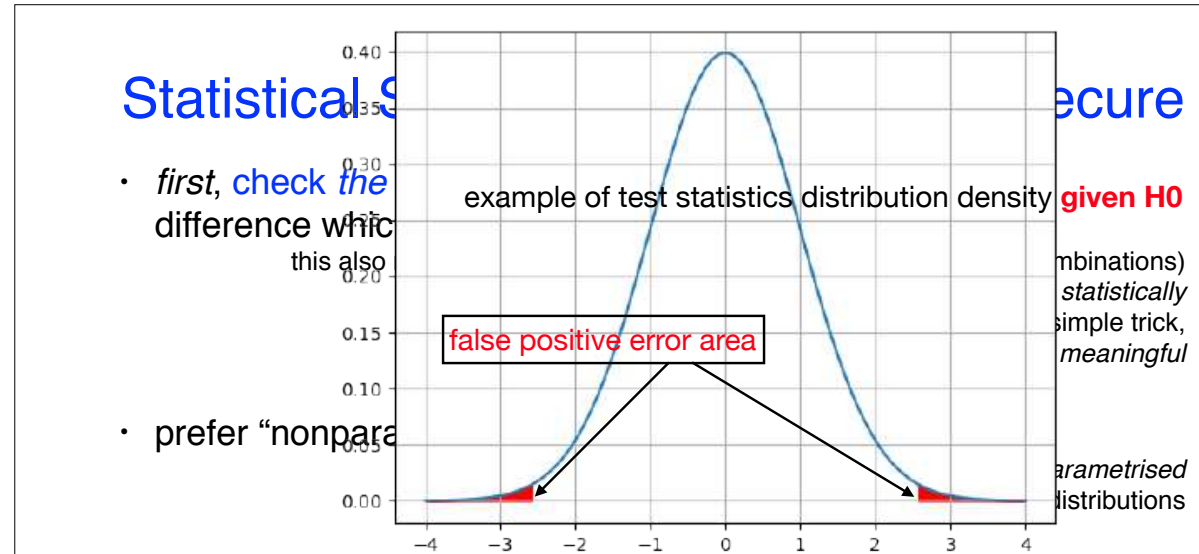

- $p$-value $=$ significance level $=$ probability of a false positive outcome, given $\mathrm{HO}$ is true

smaller $p$-values are better $<0.1 \%$ or $<1 \%$ or $<5 \%$ is usually considered as statistically significant

- given a found/observed p-value, fewer data are better

more data (almost inevitably) lead to smaller $p$-values, hence to achieve the same $p$-value with fewer data, the between-difference must be larger compared to the within-variation

\section{Statistical Significance: Methods}

- use the rank-sum test (aka Wilcoxon or Mann-Whitney U test)

- Assumption: all observations (data values) are obtained independently and no equal values are observed

The "lack" of necessary preconditions is the main reason to use the rank-sum test. even a few equal values are not detrimental

the rank-sum test is nearly as efficient as the t-test which requires normal distributions

- Null hypothesis (nothing relevant is observed if): $\operatorname{Pr}(x<y)=\operatorname{Pr}(y$ $<x)$

HO: the probability to be greater or smaller (better or worse) is the same the aim is to be able to reject the null hypothesis

- Procedure: compute the sum of ranks in the ranking of all (combined) data values

- Outcome: a $p$-value

the probability that the observed or a more extreme data set was generated under the null hypothesis; the probability to mistakenly reject the null hypothesis

\section{Statistical Significance: General Prodecure}

- first, check the relevance of the result, for example of the difference which is to be tested for statistical significance

this also means: do not explorative testing (e.g. test all pairwise combinations) any ever so small difference can be made statistically significant with a simple trick, but not made significant in the sense of important or meaningful

- prefer "nonparametric" methods

not assuming that the data come from a parametrised family of probability distributions

- $p$-value $=$ significance level $=$ probability of a false positive outcome, given $\mathrm{HO}$ is true

smaller $\mathrm{p}$-values are better

$<0.1 \%$ or $<1 \%$ or $<5 \%$ is usually considered as statistically significant

given a found/observed p-value, fewer data are better

more data (almost inevitably) lead to smaller $p$-values, hence to achieve the same $p$-value with fewer data, the between-difference must be larger compared to the within-variation 
Statistical Significance: How many data do we need?

- In the best case: at least ten (two times five) and two times nine is plenty

minimum number of data to possibly get two-sided $p<1 \%: 5+5$ or $4+6$ or $3+9$ or $2+19$ or $1+200$ and $p<5 \%: 4+4$ or $3+5$ or $2+8$ or $1+40$

- I often take two times 11 or 31 or 51

median, 5\%-tile and 95\%-tile are easily accessible with 11 or 31 or $51 \ldots$ data

- Too many data make statistical significance meaningless

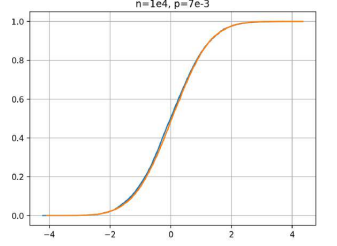

\section{Statistical Analysis}

"experimental results lacking proper statistical analysis must be considered anecdotal at best, or even wholly inaccurate"

\section{- M. Wineberg}

Do you agree (sounds about right) or disagree (is taken a little over the top) with the quote?

an experimental result (shown are all data obtained):

Do we (even) need a statistical analysis?

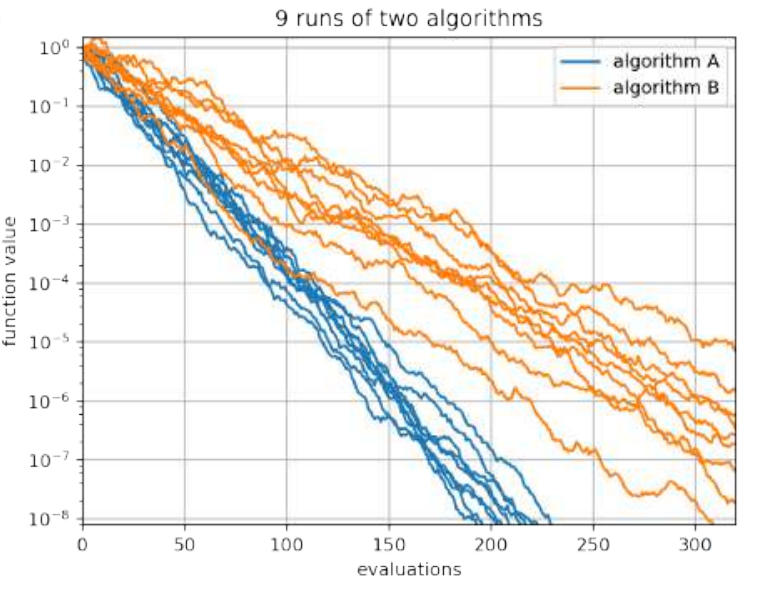

Statistical Significance: How many data do we need?

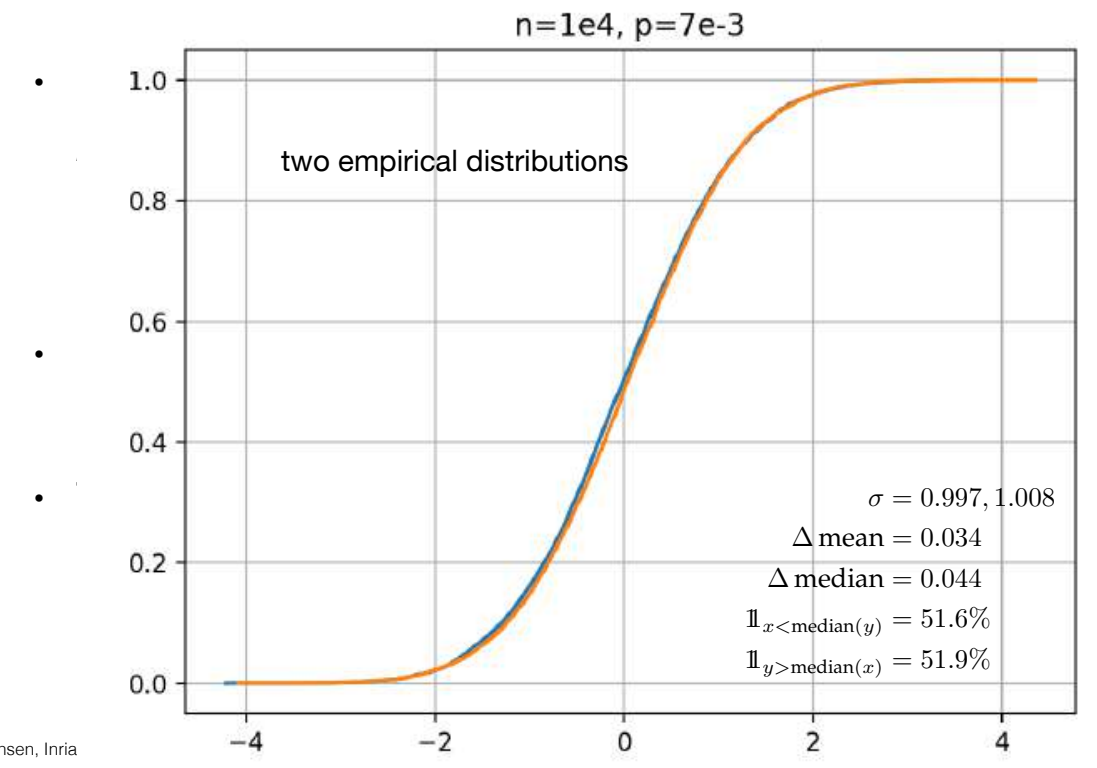

\section{Jupyter IPython notebook}
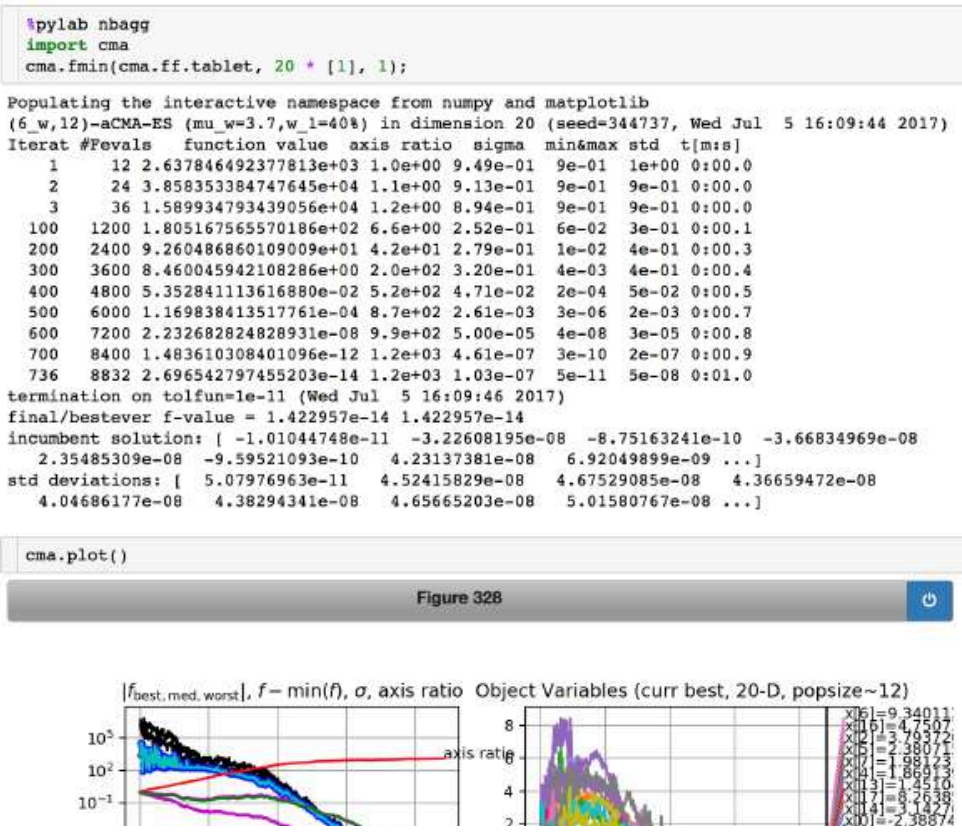


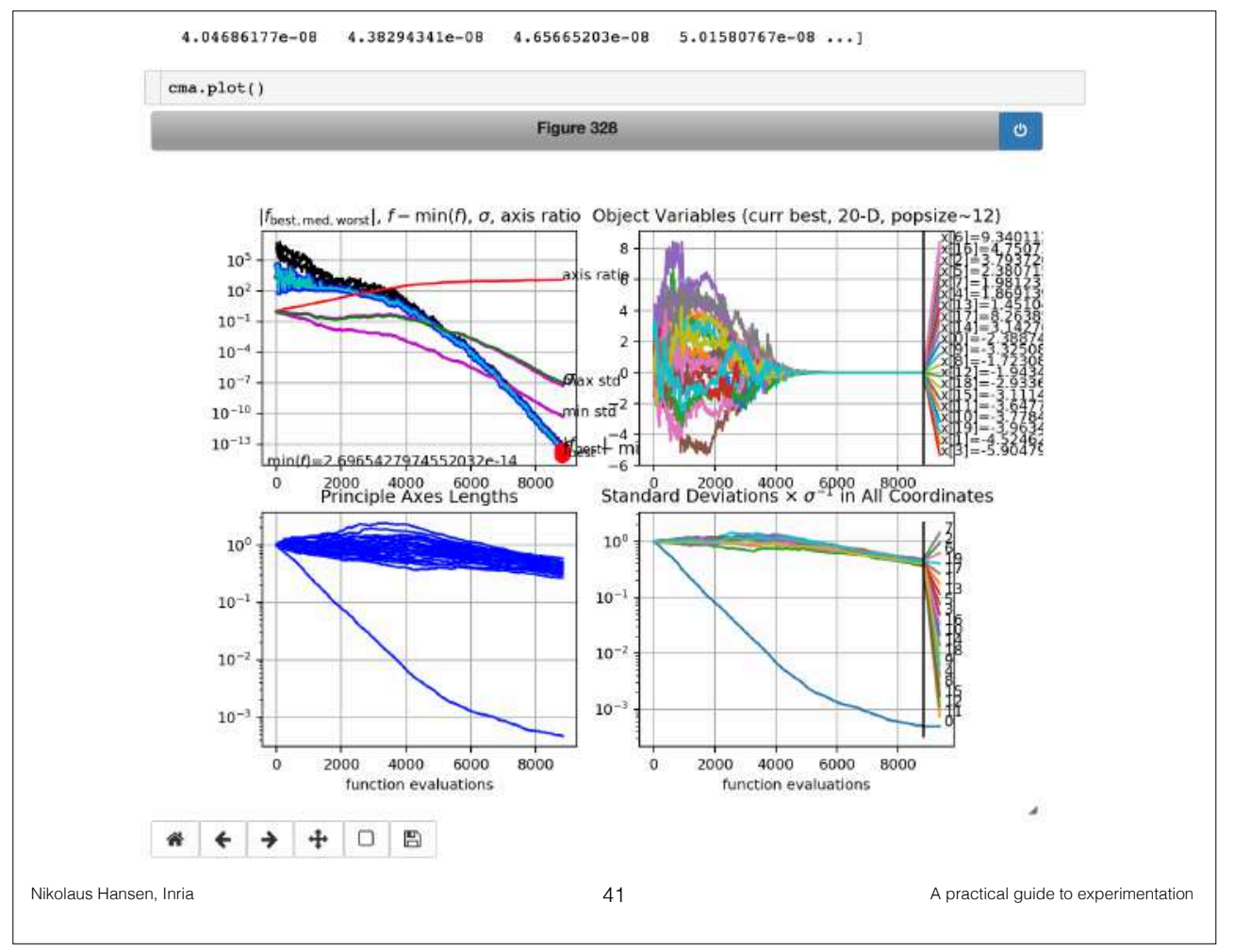

\section{Jupyter IPython notebook}

\section{- downloadsinstall anaconda python}

\# shell cmd "conda create" in case a different Python version is needed \# shell cmd "pip install cma" to install a CMA-ES module (or see github)

\# shell cmd "jupyter-notebook" and click on compact-ga.ipynb

from future_ import division, print_function

spylab nbags

Populating the interactive namespace from numpy and matplotlib

see https://github.com/nikohansen/GECCO-2019-experimentation-guide-notebooks

\section{- Demonstrations}

\section{- A somewhat typical working mode}

\section{- A parameter investigation}

\section{Questions?}

\section{Approaching an unknown problem}

- Problem/variable encoding

for example log scale vs linear scale vs quadratic transformation

- Fitness formulation

for example $\sum_{i}\left|x_{i}\right|$ and $\sum_{i} x_{i}^{2}$ have the same optimal (minimal) solution but may be very differently "optimizable".

- Create sections plots ( $\mathrm{f}$ vs $\mathrm{x}$ on a line)

one-dimensional grid search is cheap

- Try to locally improve a given (good) solution

- Start local search from different initial solutions.

Ending up always in different solutions? Or always in the same?

- Apply "global search" setting

- see also http://cma.gforge.inria.fr/cmaes sourcecode page.html\#practical 


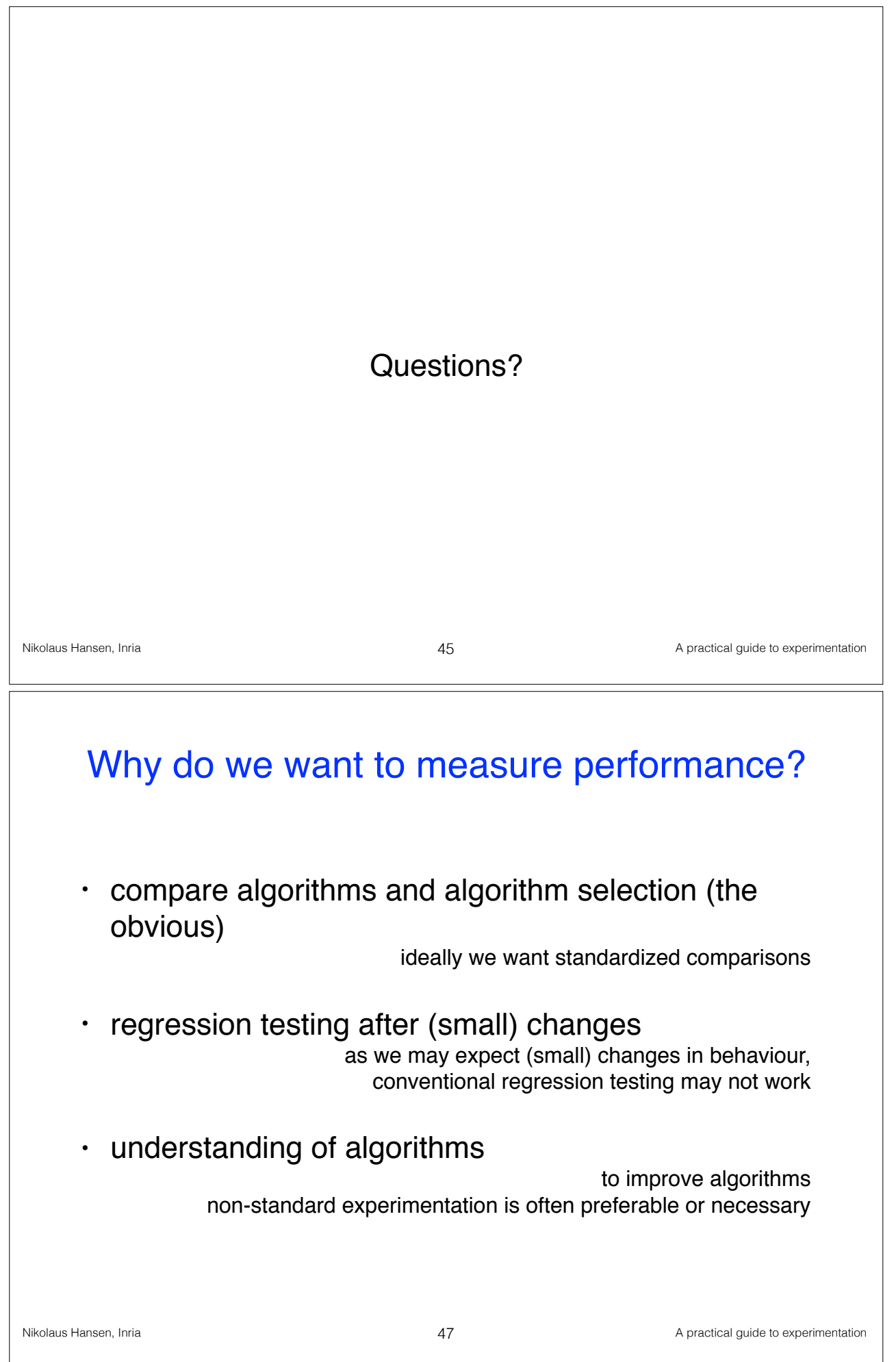

\section{Performance Assessment}

- methodology: run an algorithm on a set of test functions and extract performance measures from the generated data

choice of measure and aggregation

- display

subtle display changes can make a huge difference

- there are surprisingly many devils in the details

\section{Aggregation: Fixed Budget vs Fixed Target}

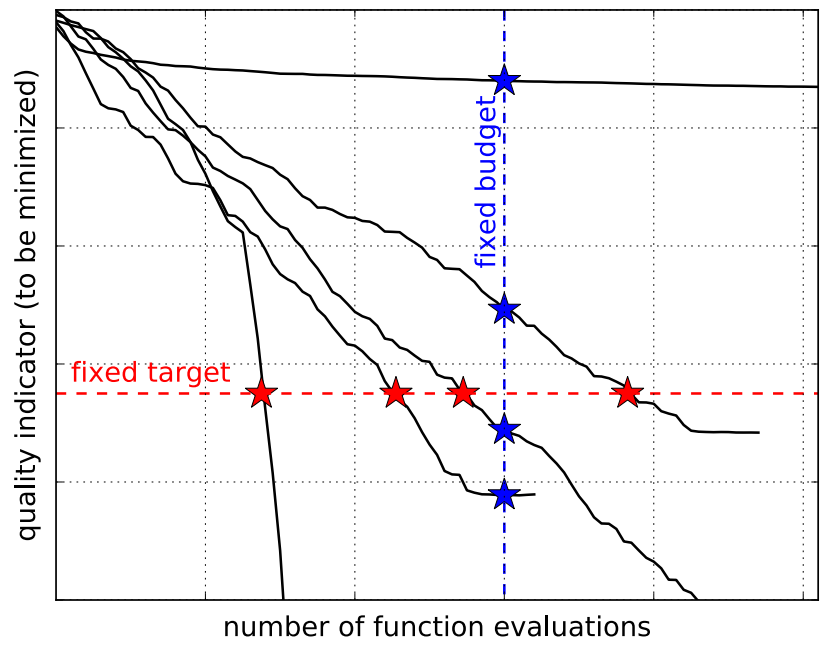

- for aggregation we need comparable data

- missing data: problematic when many runs lead to missing data - fixed target approach misses out on bad results (we may correct for this to some extend) - fixed target approach misses out on bad results (we
- fixed budget approach misses out on good results 


\section{Performance Measures for Evaluation}

Generally, a performance measure should be

- quantitative on the ratio scale (highest possible) "algorithm $\mathrm{A}$ is two times better than algorithm $\mathrm{B}$ " as "performance $(B) /$ performance $(A)=1 / 2=0.5$ " should be meaningful statements

- assuming a wide range of values

- meaningful (interpretable) with regard to the real world

transfer the measure from benchmarking to real world

runtime or first hitting time is the prime candidate

\section{Fixed Budget vs Fixed Target}

Fixed budget $=>$ measuring/display final/best $f$-values

Fixed target => measuring/display needed budgets (\#evaluations)

Number of function evaluations:

- are quantitatively comparable (on a ratio scale)

ratio scale: "A is 3.5 times faster than $B$ ", $A / B=1 / 3.5$ is a meaningful notion

- the measurement itself is interpretable independently of the function time remains the same time regardless of the underlying problem 3 times faster is 3 times faster is 3 times faster on every problem

- there is a clever way to account for missing data

$=>$ fixed target is (much) preferable

\section{Aggregation: Fixed Budget vs Fixed Target}

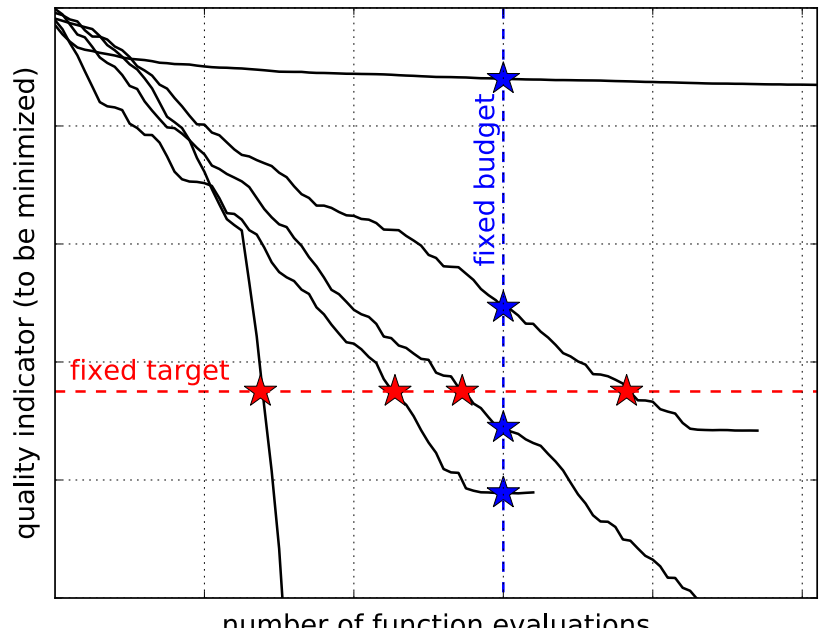

number of function evaluations

- for aggregation we need comparable data

- missing data: problematic when many runs lead to missing data - fixed target approach misses out on bad results (we may correct for this to some extend) fixed budget approach misses out on good results

\section{The Problem of Missing Values}

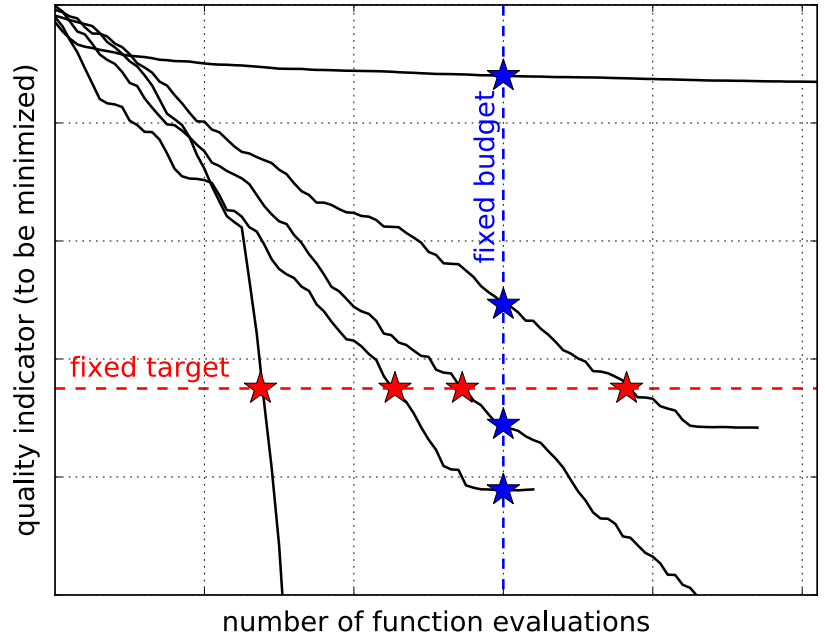

for aggregation we need comparable data

- missing data: problematic when many runs lead to missing data - fixed target approach misses out on bad results (we may correct for this to some extend) - fixed budget approach misses out on good results 
Consider simulated (artificial) restarts using the given independent runs

\section{Algo Restart A:}

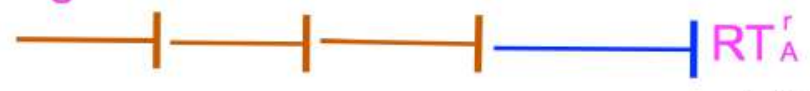

Algo Restart B:

$$
p_{s}(\text { Algo Restart A })=1
$$

$$
\mathrm{RT}_{\mathrm{B}}^{\mathrm{r}}
$$

$$
p_{s}(\text { Algo Restart } \mathrm{B})=1
$$

Caveat: the performance of algorithm A critically depends on termination methods (before to hit the target)

\section{Empirical Distribution Functions}

- Empirical cumulative distribution functions (ECDF, or in short, empirical distributions) are arguably the single most powerful tool to "aggregate" data in a display. 


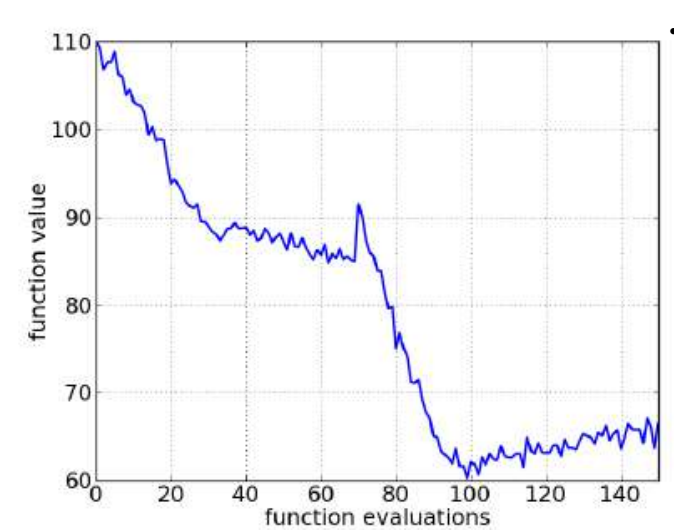

a convergence

graph

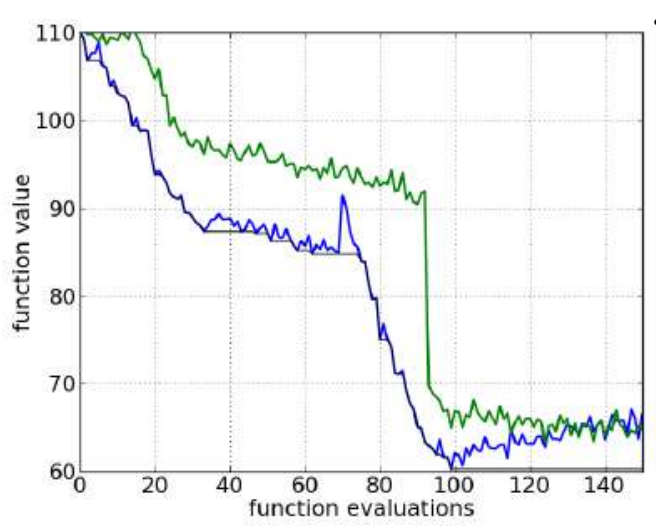

another

convergence

graph

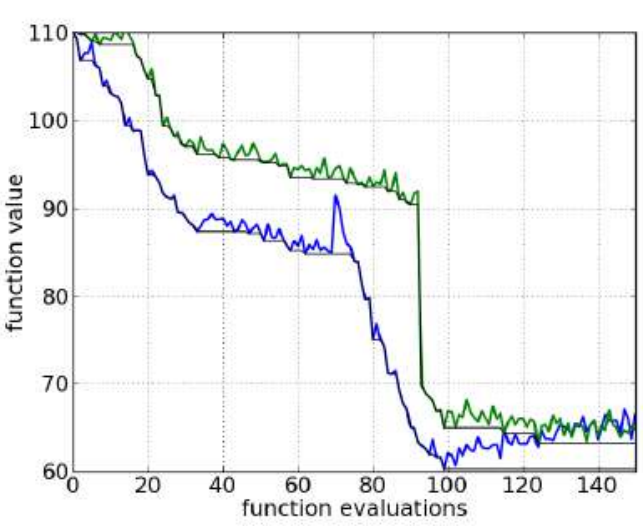

other

convergence

graph with hitting

time 


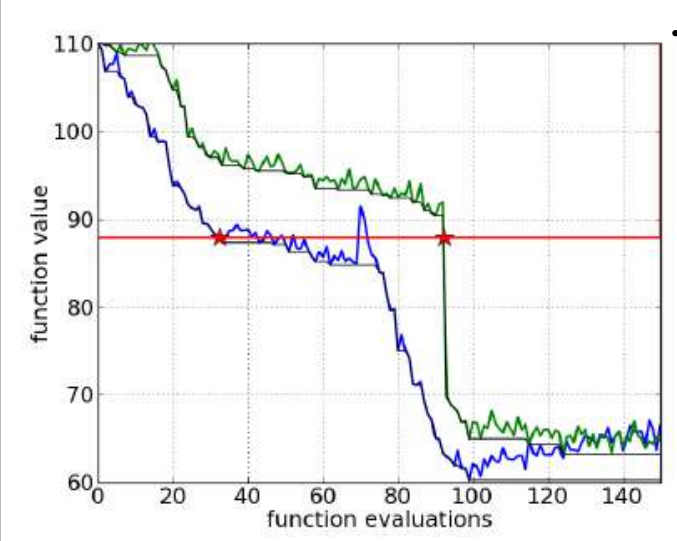

a target value

delivers two data

points (or

possibly missing

values)

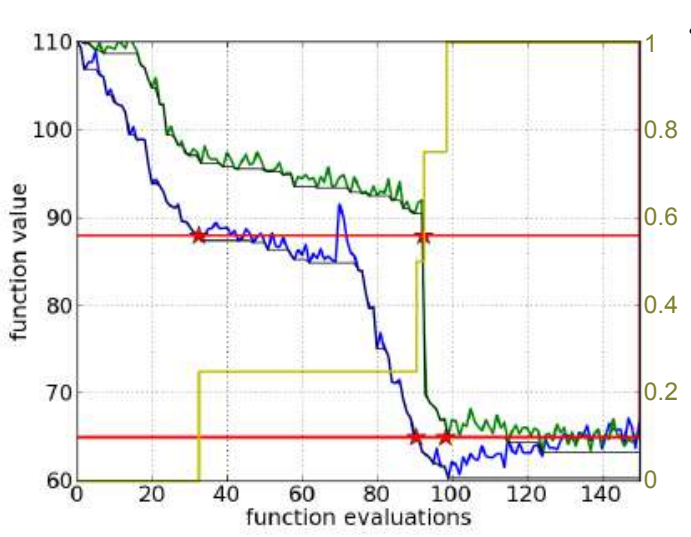

- the ECDF with

four steps

(between 0 and

1)

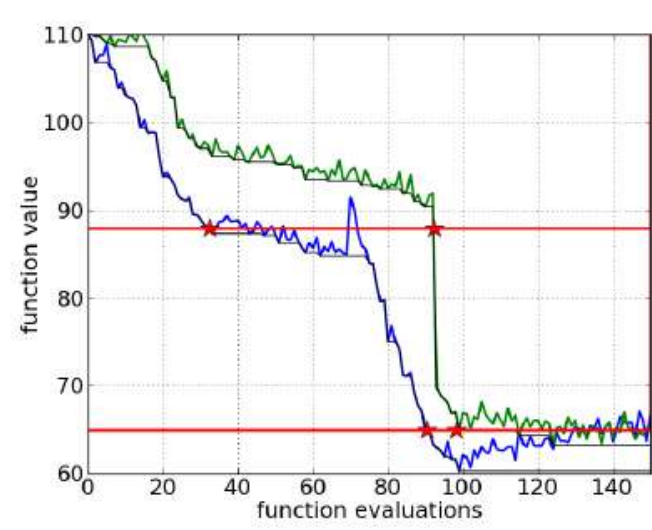

\section{another target}

value delivers

two more data

points
Nikolaus Hansen, Inria

62

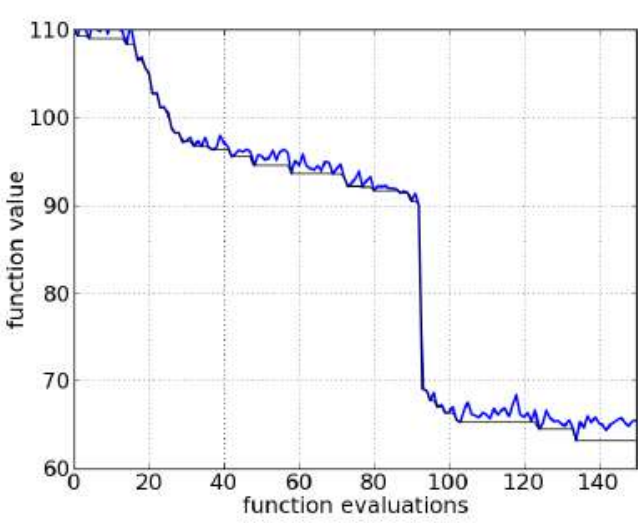

reconstructing a

single run 


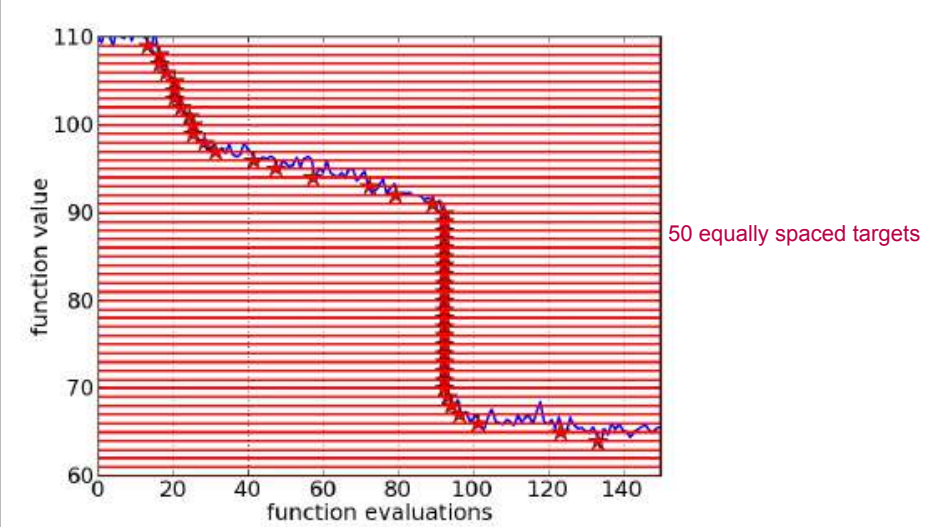

Nikolaus Hansen, Inria

65

A practical quide to experimentation

(2)

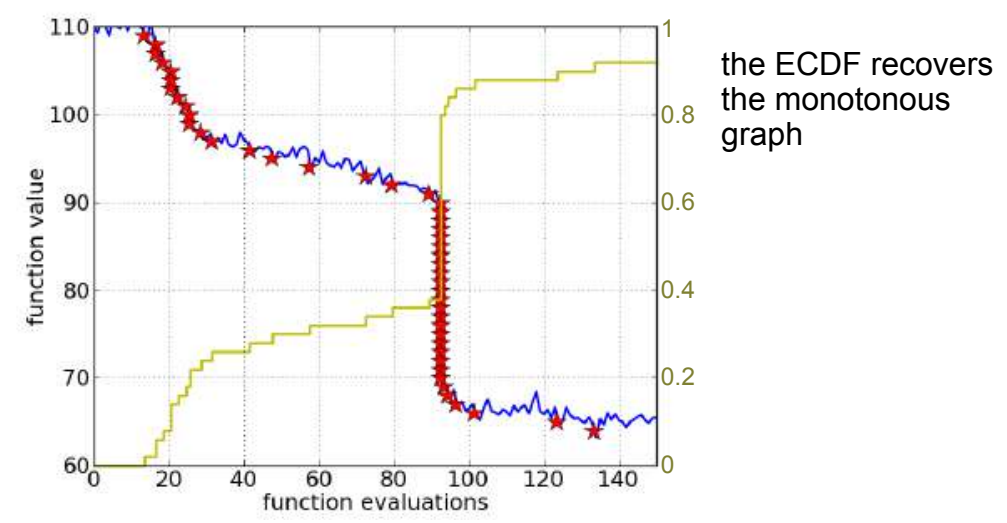

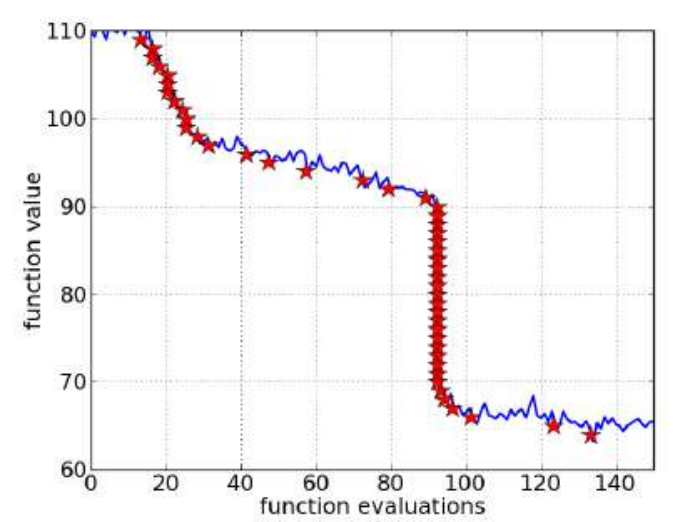

Nikolaus Hansen, Inria

66

A practical guide to experimentation

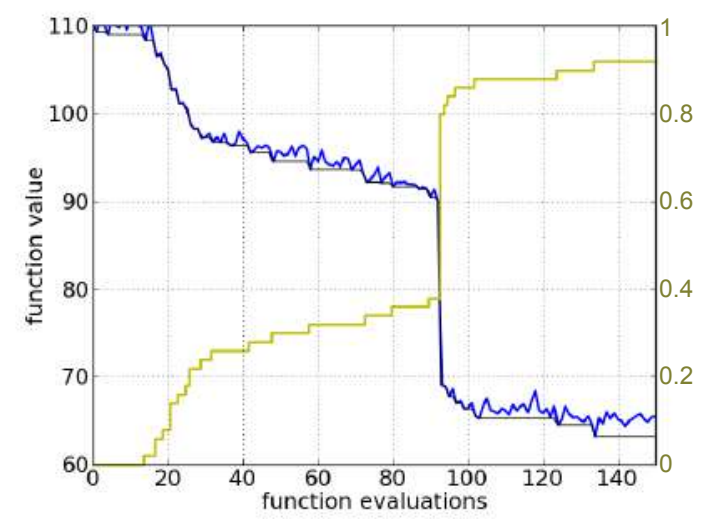

the ECDF recovers

the monotonous

graph, discretised

and flipped 


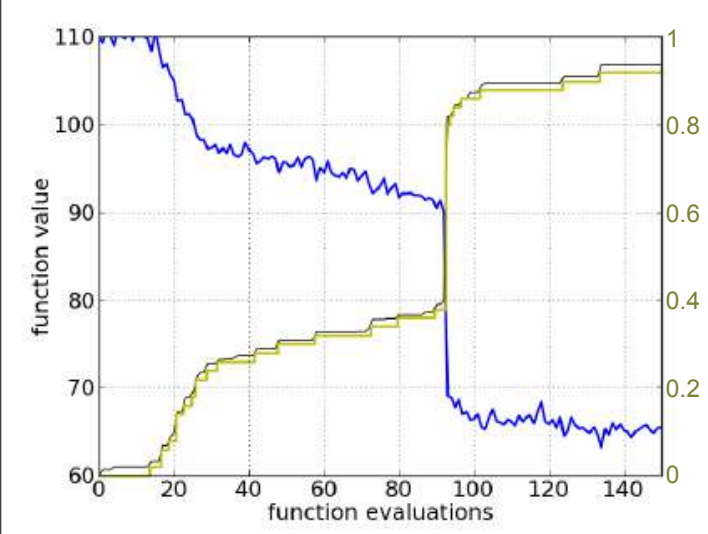

the ECDF recovers

the monotonous

graph, discretised

and flipped

\section{Data and Performance Profiles}

- so-called Data Profiles (Moré and Wild 2009) are empirical distributions of runtimes [\# evaluations] to achieve a given single target

usually divided by dimension +1

\section{- so-called Performance profiles (Dolan and Moré} 2002) are empirical distributions of relative runtimes [\# evaluations] to achieve a given single target

normalized by the runtime of the fastest algorithm on the respective problem

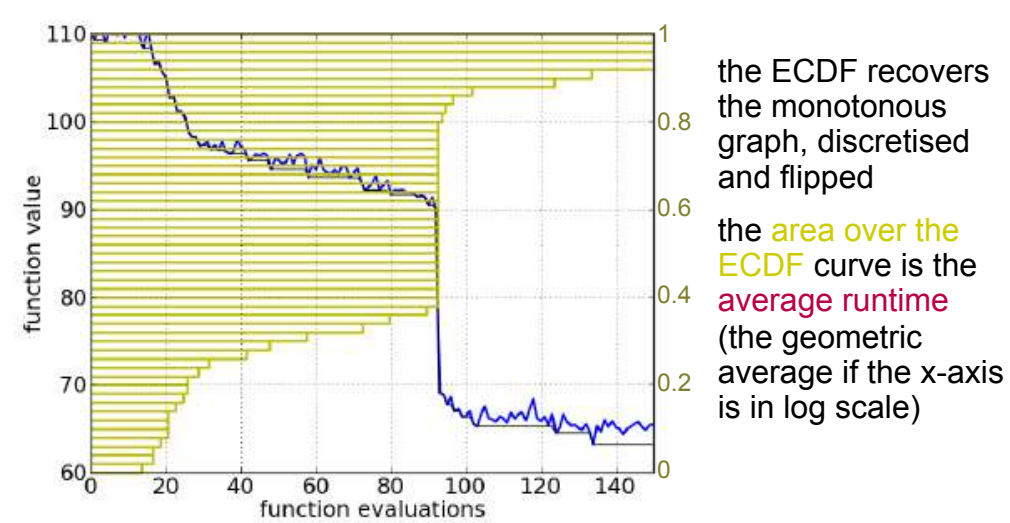

\section{Benchmarking with $\mathrm{COCO}$}

COCO - Comparing Continuous Optimisers

- is a (software) platform for comparing continuous optimisers in a black-box scenario

https://github.com/numbbo/coco

- automatises the tedious and repetitive task of benchmarking numerical optimisation algorithms in a black-box setting

- advantage: saves time and prevents common (and not so common) pitfalls

\section{COCO provides}

- experimental and measurement methodology

main decision: what is the end point of measurement

- suites of benchmark functions

single objective, bi-objective, noisy, constrained (in beta stage)

- data of already benchmarked algorithms to compare with 


\section{COCO: Installation and Benchmarking in Python}

$\$$ \#\#\# get and install the code

\$ git clone https://github.com/numbbo/coco.git \# get coco using git $\$ \mathrm{~cd}$ coco

python do.py run-python \# install python experimental module cocoex

\$ python do.py install-postprocessing \# install post-processing :-)

import os, webbrowser

from scipy.optimize import fmin

import cocoex, cocopp

\# prepare

output folder = "scipy-optimize-fmin"

suite = cocoex. Suite("bbob", " ", "n")

observer = cocoex.observer ("bbob", "result folder: " + output_folder)

\# run benchmarking

for problem in suite: \# this 100 will take several minutes

observer.observe(problem) \# generates the data for cocopp post-processing fmin(problem, problem.initial solution)

\# post-process and show data

cocopp.main(observer.result folder) \# re-run folders look like "...-001" etc webbrowser.open ("file://" + os.getcwd() + "/ppdata/index.html")

\section{The COCO Benchmarking Methodology}

- budget-free larger budget means more data to investigate
any budget is comparable termination and restarts are or become relevant

- using runtime as (almost) single performance measure measured in number of function evaluations

\section{- runtimes are aggregated}

- in empirical (cumulative) distribution functions

- by taking averages

geometric average when aggregating over different problems

\section{Benchmark Functions}

\section{should be}

- comprehensible

- difficult to defeat by "cheating"

examples: optimum in zero, separable

- scalable with the input dimension

- reasonably quick to evaluate

e.g. 12-36h for one full experiment

- reflect reality

specifically, we model well-identified difficulties encountered also in real-world problems

A practical guide to experimentation

\section{Benchmarking Results for Algorithm ALG on the bbob Suite} Home

Runtime distributions (ECDFs) per function

Runtime distributions (ECDFs) summary and function groups

Scaling with dimension for selected targets

Tables for selected targets

Runtime distribution for selected targets and f-distributions

Runtime loss ratios

Runtime distributions (ECDFs) over all targets

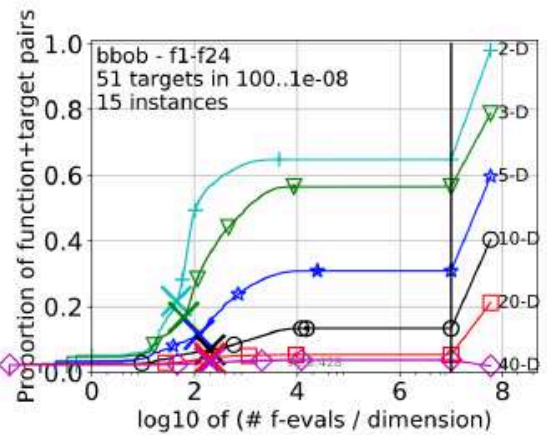

$\log 10$ of (\# f-evals / dimensi 


\section{Using Theory}

"In the course of your work, you will from time to time encounter the situation where the facts and the theory do not coincide. In such circumstances, young gentlemen, it is my earnest advice to respect the facts."

- Igor Sikorsky, airplane and helicopter designer

\section{Using Theory in Experimentation}

- shape our expectations and objectives

- debugging / consistency checks

theory may tell us what we expect to see

- knowing the limits (optimal bounds)

for example, we cannot converge faster than optima trying to improve is a waste of time

\section{- utilize invariance}

it may be possible to design a much simpler experiment and get to the same or stronger conclusion by invariance considerations change of coordinate system is a powerful tool 